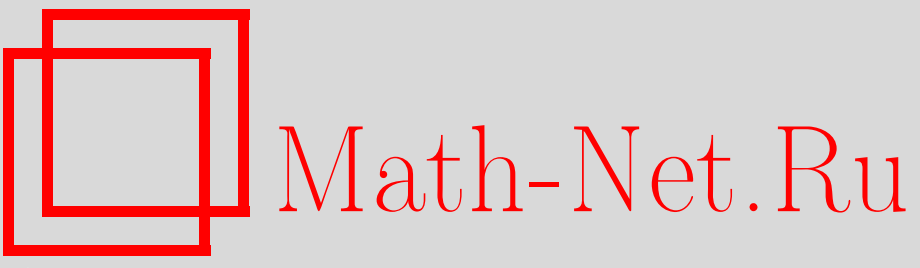

A. R. S. Juma, M. Sh. Abdul-Hussein, M. F. Hani, On a subclass of univalent functions with negative coefficients defined by a linear operator, Vestn. Udmurtsk. Univ. Mat. Mekh. Komp. Nauki, 2015, Volume 25, Issue 3, 306-317

Use of the all-Russian mathematical portal Math-Net.Ru implies that you have read and agreed to these terms of use

http://www.mathnet.ru/eng/agreement

Download details:

IP : 18.234 .197 .8

April 26, 2023, 11:41:08 
MSC: $30 \mathrm{C} 45$

(C) A.R.S. Juma, M.Sh. Abdul-Hussein, M.F. Hani

\section{ON A SUBCLASS OF UNIVALENT FUNCTIONS WITH NEGATIVE COEFFICIENTS DEFINED BY LINEAR OPERATOR}

The present paper introduces and studies the subclass $A_{n}(m, \beta, p, q, \lambda)$ of univalent functions with negative coefficients defined by new linear operator $J^{\lambda}$ in the open unit disk $\mathcal{U}=\{z \in \mathbb{C}:|z|<1\}$. The main task is to investigate several properties such as coefficient estimates, distortion theorems, closure theorems. Neighborhood and radii of starlikeness, convexity and close-to-convexity of functions belonging to the class $A_{n}(m, \beta, p, q, \lambda)$ are studied.

Keywords: analytic univalent function, Hadamard product, Ruscheweyh derivative, distortion theorems, closure theorems.

\section{$\S 1$. Introduction}

Let $A(n)$ denote the class of functions normalized by

$$
f(z)=z+\sum_{k=n+1}^{\infty} a_{k} z^{k} \quad(n \in N=1,2,3, \ldots)
$$

which are analytic and univalent in the open unit disk $\mathcal{U}=\{z \in \mathbb{C}:|z|<1\}$. Let $A^{-}(n)$ be the subclass of $A(n)$, consisting of functions of the form

$$
f(z)=z-\sum_{k=n+1}^{\infty} a_{k} z^{k} \quad\left(n \in N, a_{k} \geqslant 0\right) .
$$

For functions $f(z) \in A(n)$ given by (1.1) and $g(z) \in A(n)$ given by

$$
g(z)=z+\sum_{k=n+1}^{\infty} b_{k} z^{k} \quad(n \in N)
$$

we define the Hadamard product (or convolution) of $f(z)$ and $g(z)$ by

$$
f(z) * g(z)=z+\sum_{k=n+1}^{\infty} a_{k} b_{k} z^{k} \quad(z \in \mathcal{U}) .
$$

We define the function $\Phi(a, c ; z)$ by

$$
\Phi(a, c ; z)=z+\sum_{k=n+1}^{\infty} \frac{(a)_{k-1}}{(b)_{k-1}} z^{k} \quad(a \in R, \quad c \in R-\{0,-1,-2, \ldots\}),
$$

where $(a)_{k}$ is the Pochhammer symbol defined by

$$
(a)_{k}=\frac{\Gamma(a+k)}{\Gamma(a)}= \begin{cases}1, & \text { if } \quad k=0, \\ a(a+1)(a+2) \cdots(a+k-1), & \text { if } k \in N .\end{cases}
$$

We consider a functions $\Phi^{-}(a, c ; z)$ defined by the convolution

$$
\Phi(a, c ; z) * \Phi^{-}(a, c ; z)=\frac{z}{(1-z)^{\lambda+1}},
$$


where $\lambda>-1, z \in \mathcal{U}$. This function yields the following family of linear operators

$$
I^{\lambda}(a, c ; z) f(z)=\Phi^{-}(a, c ; z) * f(z), \quad z \in \mathcal{U},
$$

where $a, c \in-\{0,-1,-2, \ldots\}$. For a function $f \in A^{-}(n)$, it follows that for $\lambda>-1$

$$
I^{\lambda}(a, c ; z) f(z)=z-\sum_{k=n+1}^{\infty} \frac{(c)_{k-1}(\lambda+1)_{k-1}}{(1)_{k-1}(a)_{k-1}} a_{k} z^{k}
$$

is the Cho-Kown-Srivastava integral operator [11]. Further, denote by $D^{\lambda}: A(n) \rightarrow A(n)$ the Ruscheweyh derivative of order $\lambda$ defined by $[8,13,14]$.

$$
D^{\lambda} f(z)=\frac{z}{(1-z)^{\lambda+1}} * f(z)=\frac{z\left(z^{\lambda-1} f(z)\right)^{\lambda}}{\lambda !} \quad(f(z) \in A(n)),
$$

where $\lambda>-1$. Now, for $f \in A^{-}(n)$ we defined a new operator [4]

$$
J^{\lambda} f(z)=I^{\lambda}(a, c ; z) f(z) * D^{\lambda} f(z)=z-\sum_{k=n+1}^{\infty} \frac{(c)_{k-1}(\lambda+1)_{k-1}}{(1)_{k-1}(a)_{k-1}} B_{k}(\lambda) a_{k} z^{k},
$$

where $B_{k}(\lambda)=\frac{\Gamma(\lambda+k)}{\Gamma(\lambda+1)(k-1) !}, \lambda>-1, z \in \mathcal{U}$. Next, we define the following

Definition 1. Let the function $f(z)$ be of the form (1.2). Then $f(z)$ is said to be in the class $A_{n}(m, \beta, p, q, \lambda)$ if it satisfies the following criterion

$$
\left|\frac{\frac{z\left(J^{\lambda} f(z)\right)^{\prime}}{J^{\lambda} f(z)}-1}{2 p\left(\frac{z\left(J^{\lambda} f(z)\right)^{\prime}}{J^{\lambda} f(z)}-q\right)-m\left(\frac{z\left(J^{\lambda} f(z)\right)^{\prime}}{J^{\lambda} f(z)}-1\right)}\right|<\beta,
$$

where $|z|<1,0<\beta \leqslant 1, \frac{1}{2} \leqslant p \leqslant 1,0 \leqslant q \leqslant \frac{1}{2} p$, and $\frac{1}{2}<m \leqslant 1$.

Normalized, univalent analytic functions have been extensively studied by (for example) Aghalary and Kulkarni [1], Aouf [2], Khairnar and Meena More [6], Juma and Kulkarni [5].

Remark 1. We note that by specializing the parameters $m, \beta, p, q$ and $\lambda$ we have the following subclasses.

(i) If $f(z) \in A_{n}(m, \beta, p, q, \lambda)$ with $a=c=1$ and $\lambda=0$ in (1.4), then $J^{\lambda} f(z)=f(z)$.

(ii) The class $A_{n}(1,1,1,0, \lambda)$ is the class of starlike functions in $\mathcal{U}$.

(iii) The class $A_{n}(1,1,1, q, \lambda)$ is the class of starlike functions of order $q(0 \leqslant q<1)$.

(iv) The class $A_{n}\left(1, \beta, \frac{(\alpha+1)}{2}, 0, \lambda\right)$ is the class studied by Lakshminarasimhan [9].

(v) The class $A_{n}(1, \beta, p, q, \lambda)$ is the class studied by S. R Kulkarni [7].

In this paper we shall first deduce a necessary and sufficient condition for a function $f(z)$ to be in the class $A_{n}(m, \beta, p, q, \lambda)$. Then we obtain the distortion and growth theorems, closure theorems, neighborhood and radii of univalent starlikeness, convexity and close-to-convexity of order $\delta(0 \leqslant$ $\leqslant \delta<1$ ) for this functions.

\section{$\S 2$. Coefficient inequality}

Theorem 1. Let the function $f(z)$ be of the form (1.2). Then $f(z)$ is in the class $A_{n}(m, \beta, p, q, \lambda)$ if and only if

$$
\sum_{k=n+1}^{\infty}[(k-1)(1-m \beta)-2 p \beta(k-q)] \frac{(c)_{k-1}(\lambda+1)_{k-1} B_{k}(\lambda)}{(1)_{k-1}(a)_{k-1}} a_{k} \leqslant 2 p \beta(1-q),
$$

where $0<\beta \leqslant 1, \frac{1}{2} \leqslant p \leqslant 1,0 \leqslant q \leqslant \frac{1}{2} p, \frac{1}{2}<m \leqslant 1, a, c \in R-\{0,-1,-2, \ldots\}, z \in \mathcal{U}$. 
P r o o f. Suppose that (2.1) holds true. Then we find that

$$
\begin{gathered}
\left|\frac{\frac{z\left(J^{\lambda} f(z)\right)^{\prime}}{J^{\lambda} f(z)}-1}{2 p\left(\frac{z\left(J^{\lambda} f(z)\right)^{\prime}}{J^{\lambda} f(z)}-q\right)-m\left(\frac{z\left(J^{\lambda} f(z)\right)^{\prime}}{J^{\lambda} f(z)}-1\right)}\right|= \\
=\left|\frac{-\sum_{k=n+1}^{\infty}(k-1) \frac{(c)_{k-1}(\lambda+1)_{k-1} B_{k}(\lambda)}{(1)_{k-1}(a)_{k-1}} a_{k} z^{k}}{2 p(1-q) z+\sum_{k=n+1}^{\infty}[m(k-1)-2 p(k-q)] \frac{(c)_{k-1}(\lambda+1)_{k-1} B_{k}(\lambda)}{(1)_{k-1}(a)_{k-1}} a_{k} z^{k}}\right| \leqslant \\
\leqslant \frac{\sum_{k=n+1}^{\infty}(k-1) \frac{(c)_{k-1}(\lambda+1)_{k-1} B_{k}(\lambda)}{(1)_{k-1}(a)_{k-1}} a_{k}|z|^{k-1}}{2 p(1-q)-\sum_{k=n+1}^{\infty}[m(k-1)-2 p(k-q)] \frac{(c)_{k-1}(\lambda+1)_{k-1} B_{k}(\lambda)}{(1)_{k-1}(a)_{k-1}} a_{k}|z|^{k-1}}<\beta .
\end{gathered}
$$

Choosing values of $z$ on real axis and letting $z \rightarrow 1^{-}$, we have

$$
[(k-1)(1-m \beta)-2 p \beta(k-q)] \frac{(c)_{k-1}(\lambda+1)_{k-1} B_{k}(\lambda)}{(1)_{k-1}(a)_{k-1}} a_{k}-2 p \beta(1-q) \leqslant 0 .
$$

By hypothesis, thus by maximum modulus theorem, we have

$$
f(z) \in A_{n}(m, \beta, p, q, \lambda) .
$$

Conversely, assume that $f(z) \in A_{n}(m, \beta, p, q, \lambda)$, then from the definition of $f(z)$, we have

$$
\left|\frac{\frac{z\left(J^{\lambda} f(z)\right)^{\prime}}{J^{\lambda} f(z)}-1}{2 p\left(\frac{z\left(J^{\lambda} f(z)\right)^{\prime}}{J^{\lambda} f(z)}-q\right)-m\left(\frac{z\left(J^{\lambda} f(z)\right)^{\prime}}{J^{\lambda} f(z)}-1\right)}\right|<\beta,
$$

that is

$$
\left|\frac{-\sum_{k=n+1}^{\infty}(k-1) \frac{(c)_{k-1}(\lambda+1)_{k-1} B_{k}(\lambda)}{(1)_{k-1}(a)_{k-1}} a_{k} z^{k}}{2 p(1-q) z+\sum_{k=n+1}^{\infty}[m(k-1)-2 p(k-q)] \frac{(c)_{k-1}(\lambda+1)_{k-1} B_{k}(\lambda)}{(1)_{k-1}(a)_{k-1}} a_{k} z^{k}}\right|<\beta .
$$

By the fact $|\operatorname{Re}(z)| \leqslant|z|$ for all $z$, we have

$$
\operatorname{Re}\left\{\frac{\sum_{k=n+1}^{\infty}(k-1) \frac{(c)_{k-1}(\lambda+1)_{k-1} B_{k}(\lambda)}{(1)_{k-1}(a)_{k-1}} a_{k}|z|^{k-1}}{2 p(1-q) z+\sum_{k=n+1}^{\infty}[m(k-1)-2 p(k-q)] \frac{(c)_{k-1}(\lambda+1)_{k-1} B_{k}(\lambda)}{(1)_{k-1}(a)_{k-1}} a_{k}|z|^{k-1}}\right\}<\beta .
$$

We choose the values of $z$ on real axis such that $\frac{\left(J^{\lambda} f(z)\right)^{\prime}}{J^{\lambda} f(z)}$ is real and upon clearing, the denominator of the above expression and allowing $z \rightarrow 1^{-}$through real values, we obtain

$$
\sum_{k=n+1}^{\infty}[(k-1)(1-m \beta)-2 p \beta(k-q)] \frac{(c)_{k-1}(\lambda+1)_{k-1} B_{k}(\lambda)}{(1)_{k-1}(a)_{k-1}} a_{k} \leqslant 2 p \beta(1-q) .
$$

Corollary 1. Let the function $f(z)$ of the form (1.2) be in the class $A_{n}(m, \beta, p, q, \lambda)$. Then

$$
a_{k} \leqslant \frac{2 p \beta(1-q)(1)_{k-1}(a)_{k-1}}{[(k-1)(1-m \beta)-2 p \beta(k-q)](c)_{k-1}(\lambda+1)_{k-1} B_{k}(\lambda)} \quad(k \geqslant n+1, n \in N),
$$

where $0<\beta \leqslant 1, \frac{1}{2} \leqslant p \leqslant 1,0 \leqslant q \leqslant \frac{1}{2} p, \frac{1}{2}<m \leqslant 1, a, c \in R-\{0,-1,-2, \ldots\}, z \in \mathcal{U}$.

Remark 2. If $f(z) \in A_{n}(1, \beta, p, q, \lambda)$, then

$$
a_{k} \leqslant \frac{2 p \beta(1-q)(1)_{k-1}(a)_{k-1}}{[(k-1)(1-\beta)-2 p \beta(k-q)](c)_{k-1}(\lambda+1)_{k-1} B_{k}(\lambda)} \quad(k \geqslant n+1, n \in N)
$$

and equality holds for

$$
f(z)=z-\frac{2 p \beta(1-q)(1)_{k-1}(a)_{k-1}}{[(k-1)(1-\beta)-2 p \beta(k-q)](c)_{k-1}(\lambda+1)_{k-1} B_{k}(\lambda)} z^{k} .
$$


Remark 3. If $f(z) \in A_{n}(1, \beta, 1, q, \lambda)$, then

$$
a_{k} \leqslant \frac{2 \beta(1-q)(1)_{k-1}(a)_{k-1}}{[(k-1)(1-\beta)-2 \beta(k-q)](c)_{k-1}(\lambda+1)_{k-1} B_{k}(\lambda)} \quad(k \geqslant n+1, n \in N)
$$

and equality holds for

$$
f(z)=z-\frac{2 \beta(1-q)(1)_{k-1}(a)_{k-1}}{[(k-1)(1-\beta)-2 \beta(k-q)](c)_{k-1}(\lambda+1)_{k-1} B_{k}(\lambda)} z^{k} .
$$

Remark 4. If $f(z) \in A_{n}(1,1,1, q, \lambda)$, then

$$
a_{k} \leqslant \frac{-(1-q)(1)_{k-1}(a)_{k-1}}{(k-q)(c)_{k-1}(\lambda+1)_{k-1} B_{k}(\lambda)} \quad(k \geqslant n+1, n \in N)
$$

and equality holds for

$$
f(z)=z+\frac{(1-q)(1)_{k-1}(a)_{k-1}}{(k-q)(c)_{k-1}(\lambda+1)_{k-1} B_{k}(\lambda)} z^{k}
$$

\section{$\S 3$. Distortion theorems}

Theorem 2. Let the function $f(z)$ of the form (1.2) be in the class $A_{n}(m, \beta, p, q, \lambda)$. Then for $|z|=r<1$, we have

$$
\begin{aligned}
& r-\frac{2 p \beta(1-q)(1)_{n}(a)_{n}}{[n(1-m \beta)-2 p \beta(n+1-q)](c)_{n}(\lambda+1)_{n} B_{n+1}(\lambda)} r^{n+1} \leqslant|f(z)| \leqslant \\
& \quad \leqslant r+\frac{2 p \beta(1-q)(1)_{n}(a)_{n}}{[n(1-m \beta)-2 p \beta(n+1-q)](c)_{n}(\lambda+1)_{n} B_{n+1}(\lambda)} r^{n+1} .
\end{aligned}
$$

The result is sharp for the function $f(z)$ given by

$$
f(z)=z-\frac{2 p \beta(1-q)(1)_{n}(a)_{n}}{[n(1-m \beta)-2 p \beta(n+1-q)](c)_{n}(\lambda+1)_{n} B_{n+1}(\lambda)} z^{n+1} .
$$

P r o o f. Suppose that $f(z) \in A_{n}(m, \beta, p, q, \lambda)$. By the inequality (2.1), since

$$
[n(1-m \beta)-2 p \beta(n+1-q)] \frac{(c)_{n}(\lambda+1)_{n}}{(1)_{n}(a)_{n}} B_{n+1}(\lambda)
$$

is non decreasing and positive for $k \geqslant n+1$, we have

$$
\begin{gathered}
{[n(1-m \beta)-2 p \beta(n+1-q)] \frac{(c)_{n}(\lambda+1)_{n}}{(1)_{n}(a)_{n}} B_{n+1}(\lambda) \sum_{k=n+1}^{\infty} a_{k} \leqslant} \\
\leqslant \sum_{k=n+1}^{\infty}[(k-1)(1-m \beta)-2 p \beta(k-q)] \frac{(c)_{k-1}(\lambda+1)_{k-1}}{(1)_{k-1}(a)_{k-1}} B_{k}(\lambda) a_{k} \leqslant 2 p \beta(1-q) .
\end{gathered}
$$

This is equivalent to

$$
\sum_{k=n+1}^{\infty} a_{k} \leqslant \frac{2 p \beta(1-q)(1)_{n}(a)_{n}}{[n(1-m \beta)-2 p \beta(n+1-q)](c)_{n}(\lambda+1)_{n} B_{n+1}(\lambda)} .
$$

Using (1.2) and (3.1), we obtain

$$
f(z)=z-\sum_{k=n+1}^{\infty} a_{k} z^{k}
$$




$$
\begin{gathered}
|f(z)| \geqslant|z|-\sum_{k=n+1}^{\infty} a_{k}|z|^{k} \geqslant r-\sum_{k=n+1}^{\infty} a_{k} r^{k} \geqslant r-r^{n+1} \sum_{k=n+1}^{\infty} a_{k} \geqslant \\
\geqslant r-\frac{2 p \beta(1-q)(1)_{n}(a)_{n}}{[n(1-m \beta)-2 p \beta(n+1-q)](c)_{n}(\lambda+1)_{n} B_{n+1}(\lambda)} r^{n+1} .
\end{gathered}
$$

Similarly

$$
|f(z)| \leqslant r+\frac{2 p \beta(1-q)(1)_{n}(a)_{n}}{[n(1-m \beta)-2 p \beta(n+1-q)](c)_{n}(\lambda+1)_{n} B_{n+1}(\lambda)} r^{n+1} .
$$

This completes the proof of Theorem 2 .

Theorem 3. Let the function $f(z)$ of the form (1.2) be in the class $A_{n}(m, \beta, p, q, \lambda)$. Then for $|z|=r<1$, we have

$$
\begin{gathered}
1-\frac{2 p \beta(1+n)(1-q)(1)_{n}(a)_{n}}{[n(1-m \beta)-2 p \beta(n+1-q)](c)_{n}(\lambda+1)_{n} B_{n+1}(\lambda)} r^{n} \leqslant\left|f^{\prime}(z)\right| \leqslant \\
\leqslant 1+\frac{2 p \beta(1+n)(1-q)(1)_{n}(a)_{n}}{[n(1-m \beta)-2 p \beta(n+1-q)](c)_{n}(\lambda+1)_{n} B_{n+1}(\lambda)} r^{n} .
\end{gathered}
$$

The result is sharp for the function $f(z)$ given by

$$
f(z)=z-\frac{2 p \beta(1+n)(1-q)(1)_{n}(a)_{n}}{[n(1-m \beta)-2 p \beta(n+1-q)](c)_{n}(\lambda+1)_{n} B_{n+1}(\lambda)} z^{n+1} .
$$

P r o o f. From (1.2) and (3.1) we have $f^{\prime}(z)=1-\sum_{k=n+1}^{\infty} k a_{k} z^{k-1}$,

$$
\begin{aligned}
\left|f^{\prime}(z)\right| & \geqslant 1-\sum_{k=n+1}^{\infty} k a_{k}|z|^{k-1} \geqslant 1-\sum_{k=n+1}^{\infty} a_{k} r^{k-1} \geqslant 1-r^{n} \sum_{k=n+1}^{\infty} k a_{k} \geqslant \\
& \geqslant 1-\frac{2 p \beta(1+n)(1-q)(1)_{n}(a)_{n}}{[n(1-m \beta)-2 p \beta(n+1-q)](c)_{n}(\lambda+1)_{n} B_{n+1}(\lambda)} r^{n} .
\end{aligned}
$$

Similarly

$$
|f(z)| \leqslant 1+\frac{2 p \beta(1+n)(1-q)(1)_{n}(a)_{n}}{[n(1-m \beta)-2 p \beta(n+1-q)](c)_{n}(\lambda+1)_{n} B_{n+1}(\lambda)} r^{n} .
$$

This completes the proof of Theorem 3 .

\section{$\S 4$. Closure theorem}

Theorem 4. Let the functions $f_{j}(j=1,2, \ldots, s)$ defined by

$$
f_{j}(z)=z-\sum_{k=n+1}^{\infty} a_{k, j} z^{k} \quad\left(a_{k, j} \geqslant 0\right)
$$

are in the class $A_{n}(m, \beta, p, q, \lambda)$. Then the function $h(z)$ defined by $h(z)=\sum_{j=1}^{s} \mu_{j} f_{j}(z) \quad\left(\mu_{j} \geqslant 0\right)$ is also in the class $A_{n}(m, \beta, p, q, \lambda)$ if $\sum_{j=1}^{s} \mu_{j}=1$.

P r o o f. We can write

$$
h(z)=\sum_{j=1}^{s} \mu_{j}\left(z-\sum_{k=n+1}^{\infty} a_{k, j} z^{k}\right)=\sum_{j=1}^{s} \mu_{j} z-\sum_{j=1}^{s} \sum_{k=n+1}^{\infty} \mu_{j} a_{k, j} z^{k}=z-\sum_{k=n+1}^{\infty} \sum_{j=1}^{s} \mu_{j} a_{k, j} z^{k} .
$$


Furthermore, since the functions $f_{j}(z)(j=1,2, \ldots, s)$ are in the class $A_{n}(m, \beta, p, q, \lambda)$, then from Theorem 1 we have

$$
\sum_{k=n+1}^{\infty}[(k-1)(1-m \beta)-2 p \beta(k-q)] \frac{(c)_{k-1}(\lambda+1)_{k-1} B_{k}(\lambda)}{(1)_{k-1}(a)_{k-1}} a_{k, j} \leqslant 2 p \beta(1-q) .
$$

Thus it is enough to prove that

$$
\begin{aligned}
& \sum_{k=n+1}^{\infty}[(k-1)(1-m \beta)-2 p \beta(k-q)] \frac{(c)_{k-1}(\lambda+1)_{k-1} B_{k}(\lambda)}{(1)_{k-1}(a)_{k-1}}\left(\sum_{j=1}^{s} \mu_{j} a_{k, j}\right)= \\
= & \sum_{j=1}^{s} \mu_{j} \sum_{k=n+1}^{\infty}[(k-1)(1-m \beta)-2 p \beta(k-q)] \frac{(c)_{k-1}(\lambda+1)_{k-1} B_{k}(\lambda)}{(1)_{k-1}(a)_{k-1}} a_{k, j} \leqslant \\
\leqslant & \sum_{j=1}^{s} \mu_{j} 2 p \beta(1-q)=2 p \beta(1-q) .
\end{aligned}
$$

Hence the proof is complete.

Corollary 2. Let the functions $f_{j}(j=1,2)$ defined by (4.1) are in the class $A_{n}(m, \beta, p, q, \lambda)$. Then the function $h(z)$ defined by $h(z)=(1-\zeta) f_{1}(z)+\zeta f_{2}(z), 0 \leqslant \zeta \leqslant 1$, is also in the class $A_{n}(m, \beta, p, q, \lambda)$.

\section{$\S 5$. Extreme points}

Theorem 5. Let $f_{1}(z)=z$ and

$$
f_{k}(z)=z-\frac{2 p \beta(1-q)(1)_{k-1}(a)_{k-1}}{[(k-1)(1-m \beta)-2 p \beta(k-q)](c)_{k-1}(\lambda+1)_{k-1} B_{k}(\lambda)} z^{k} \quad(k \geqslant n+1, n \in N) .
$$

Then the function $f(z)$ of the form (1.2) is in the class $A_{n}(m, \beta, p, q, \lambda)$ if and only if it can be expressed in the form

$$
f(z)=\mu_{1} f_{1}(z)+\sum_{k=n+1}^{\infty} \mu_{k} f_{k}(z)
$$

where $\mu_{1} \geqslant 0, \mu_{k} \geqslant 0, k \geqslant n+1$, and $\mu_{1}+\sum_{k=n+1}^{\infty} \mu_{k}=1$.

P r o o f. Assume that $f(z)$ can be expressed in the form $f(z)=\mu_{1} f_{1}(z)+\sum_{k=n+1}^{\infty} \mu_{k} f_{k}(z)$,

$$
f(z)=z-\sum_{k=n+1}^{\infty} \frac{2 p \beta(1-q)(1)_{k-1}(a)_{k-1}}{[(k-1)(1-m \beta)-2 p \beta(k-q)](c)_{k-1}(\lambda+1)_{k-1} B_{k}(\lambda)} \mu_{k} z^{k} .
$$

Thus

$$
\begin{gathered}
\sum_{k=n+1}^{\infty} \frac{[(k-1)(1-m \beta)-2 p \beta(k-q)](c)_{k-1}(\lambda+1)_{k-1} B_{k}(\lambda)}{2 p \beta(1-q)(1)_{k-1}(a)_{k-1}} \\
\cdot \sum_{k=n+1}^{\infty} \frac{2 p \beta(1-q)(1)_{k-1}(a)_{k-1}}{[(k-1)(1-m \beta)-2 p \beta(k-q)](c)_{k-1}(\lambda+1)_{k-1} B_{k}(\lambda)} \mu_{k}=\sum_{k=n+1}^{\infty} \mu_{k}=1-\mu_{1} \leqslant 1 .
\end{gathered}
$$

Hence $f(z) \in A_{n}(m, \beta, p, q, \lambda)$.

Conversely, assume that $f(z) \in A_{n}(m, \beta, p, q, \lambda)$. Setting

$$
\mu_{k}=\frac{[(k-1)(1-m \beta)-2 p \beta(k-q)](c)_{k-1}(\lambda+1)_{k-1} B_{k}(\lambda)}{2 p \beta(1-q)(1)_{k-1}(a)_{k-1}} a_{k}
$$

since $\mu_{1}=1-\sum_{k=n+1}^{\infty} \mu_{k}$. Thus $f(z)=\mu_{1} f_{1}(z)+\sum_{k=n+1}^{\infty} \mu_{k} f_{k}(z)$. Hence the proof is complete. 
Corollary 3. The extreme points of the class $A_{n}(m, \beta, p, q, \lambda)$ are the functions $f_{1}(z)=z$ and

$$
f_{k}(z)=z-\frac{2 p \beta(1-q)(1)_{k-1}(a)_{k-1}}{[(k-1)(1-m \beta)-2 p \beta(k-q)](c)_{k-1}(\lambda+1)_{k-1} B_{k}(\lambda)} z^{k} \quad(k \geqslant n+1, n \in N) .
$$

\section{$\S 6$. Neighborhood property}

The concept of neighborhood of analytic function was first introduced by Goodman [3]. Ruscheweyh [14], Raina and Srivastava [12], Orhan and Kamali [10], Silverman [15] investigated this concept for the elements of several famous subclass of analytic functions. We define the $(n, \delta)$ neighborhood of a function $f(z) \in A^{-}(n)$ by

$$
N_{n, \delta}(f)=\left\{g \in A^{-}(n): g(z)=z-\sum_{k=n+1}^{\infty} b_{k} z^{k} \text { and } \sum_{k=n+1}^{\infty} k\left|a_{k}-b_{k}\right| \leqslant \delta\right\} .
$$

In particular, for $e(z)=z$

$$
N_{n, \delta}(e)=\left\{g \in A^{-}(n): g(z)=z-\sum_{k=n+1}^{\infty} b_{k} z^{k} \text { and } \sum_{k=n+1}^{\infty} k\left|b_{k}\right| \leqslant \delta\right\} .
$$

Furthermore, a function of the form (1.2) is said to be in the class $A_{n}^{\sigma}(m, \beta, p, q, \lambda)$ if there exists a function $h(z) \in A_{n}(m, \beta, p, q, \lambda)$ such that

$$
\left|\frac{f(z)}{h(z)}-1\right|<1-\sigma \quad(z \in \mathcal{U}, 0 \leqslant \sigma<1)
$$

Theorem 6. If

$$
\frac{(c)_{k-1}(\lambda+1)_{k-1}}{(1)_{k-1}(a)_{k-1}} B_{k}(\lambda) \geqslant \frac{(c)_{n}(\lambda+1)_{n}}{(1)_{n}(a)_{n}} B_{n+1}(\lambda) \quad(k \geqslant n+1, n \in N)
$$

and

$$
\delta=\frac{2 p \beta(1-q)(1)_{n}(a)_{n}}{[(n)(1-m \beta)-2 p \beta(n+1-q)](c)_{n}(\lambda+1)_{n} B_{n+1}(\lambda)},
$$

then $A_{n}(m, \beta, p, q, \lambda) \in N_{n, \delta}(e)$.

P r o o f. Let $f(z) \in A_{n}(m, \beta, p, q, \lambda)$. Then in view of assertion (2.1) of Theorem 1 and the condition (6.4), we get

$$
\begin{gathered}
{[n(1-m \beta)-2 p \beta(n+1-q)] \frac{(c)_{n}(\lambda+1)_{n}}{(1)_{n}(a)_{n}} B_{n+1}(\lambda) \sum_{k=n+1}^{\infty} a_{k} \leqslant} \\
\leqslant \sum_{k=n+1}^{\infty}[(k-1)(1-m \beta)-2 p \beta(k-q)] \frac{(c)_{k-1}(\lambda+1)_{k-1}}{(1)_{k-1}(a)_{k-1}} B_{k}(\lambda) a_{k} \leqslant 2 p \beta(1-q),
\end{gathered}
$$

which implies

$$
\sum_{k=n+1}^{\infty} a_{k} \leqslant \frac{2 p \beta(1-q)(1)_{n}(a)_{n}}{[n(1-m \beta)-2 p \beta(n+1-q)](c)_{n}(\lambda+1)_{n} B_{n+1}(\lambda)} .
$$

Appling assertion (2.1) of Theorem 1 in conjunction with (6.5), we obtain

$$
[n(1-m \beta)-2 p \beta(n+1-q)] \frac{(c)_{n}(\lambda+1)_{n}}{(1)_{n}(a)_{n}} B_{n+1}(\lambda) \sum_{k=n+1}^{\infty} a_{k} \leqslant 2 p \beta(1-q),
$$




$$
\begin{gathered}
(n+1)[n(1-m \beta)-2 p \beta(n+1-q)] \frac{(c)_{n}(\lambda+1)_{n}}{(1)_{n}(a)_{n}} B_{n+1}(\lambda) \sum_{k=n+1}^{\infty} a_{k} \leqslant 2 p \beta(n+1)(1-q), \\
\sum_{k=n+1}^{\infty} k a_{k} \leqslant \frac{2 p \beta(n+1)(1-q)(1)_{n}(a)_{n}}{[(n)(1-m \beta)-2 p \beta(n+1-q)](c)_{n}(\lambda+1)_{n} B_{n+1}(\lambda)}=\delta .
\end{gathered}
$$

By virtue of (6.1), we have $f(z) \in N_{n, \delta}(e)$. This completes the proof of Theorem 6 .

Theorem 7. If $h(z) \in A_{n}(m, \beta, p, q, \lambda)$ and

$$
\sigma=1-\frac{\delta}{(n+1)} \frac{[n(1-m \beta)-2 p \beta(n+1-q)](c)_{n}(\lambda+1)_{n} B_{n+1}(\lambda)}{[n(1-m \beta)-2 p \beta(n+1-q)](c)_{n}(\lambda+1)_{n} B_{n+1}(\lambda)-2 p \beta(1-q)(1)_{n}(a)_{n}},
$$

then

$$
N_{n, \delta}(h) \subset A_{n}^{\sigma}(m, \beta, p, q, \lambda) .
$$

P r o o f. Suppose that $f \in N_{n, \delta}(h)$, we then find from (1.2) that

$$
\sum_{k=n+1}^{\infty} k\left|a_{k}-b_{k}\right| \leqslant \delta
$$

which readily implies the following coefficient inequality

$$
\sum_{k=n+1}^{\infty}\left|a_{k}-b_{k}\right| \leqslant \frac{\delta}{n+1} \quad(n \in N) .
$$

Next, since $h \in A_{n}(m, \beta, p, q, \lambda)$ in the view of (6.5), we have

$$
\sum_{k=n+1}^{\infty} b_{k} \leqslant \frac{2 p \beta(1-q)(1)_{n}(a)_{n}}{[(n)(1-m \beta)-2 p \beta(n+1-q)](c)_{n}(\lambda+1)_{n} B_{n+1}(\lambda)} .
$$

Using (6.7) and (6.8), we get

$$
\begin{gathered}
\left|\frac{f(z)}{h(z)}-1\right| \leqslant \frac{\sum_{k=n+1}^{\infty}\left|a_{k}-b_{k}\right|}{1-\sum_{k=n+1}^{\infty} b_{k}} \leqslant \frac{\delta}{(n+1)\left(1-\frac{2 p \beta(1-q)(1)_{n}(a)_{n}}{[(n)(1-m \beta)-2 p \beta(n+1-q)](c)_{n}(\lambda+1)_{n} B_{n+1}(\lambda)}\right)} \leqslant \\
\leqslant \frac{\delta}{(n+1)}\left(\frac{[n(1-m \beta)-2 p \beta(n+1-q)](c)_{n}(\lambda+1)_{n} B_{n+1}(\lambda)}{[n(1-m \beta)-2 p \beta(n+1-q)](c)_{n}(\lambda+1)_{n} B_{n+1}(\lambda)-2 p \beta(1-q)(1)_{n}(a)_{n}}\right)=1-\sigma,
\end{gathered}
$$

provided that $\sigma$ is given by (6.6), thus by condition (6.3), $f \in A^{\sigma}(m, \beta, p, q, \lambda)$.

\section{$\S 7$. Radii of starlikeness, convexity and close-to-convexity}

Theorem 8. Let the function $f(z)$ of the form (1.2) be in the class $A_{n}(m, \beta, p, q, \lambda)$. Then $f$ is univalent starlike of order $\delta(0 \leqslant \delta<1)$ in $|z|<r_{1}$, where

$$
r_{1}=\inf _{k}\left\{\frac{(1-\delta)[(k-1)(1-m \beta)-2 p \beta(k-q)](c)_{k-1}(\lambda+1)_{k-1} B_{k}(\lambda)}{2 p \beta(1-q)(k-\delta)(1)_{k-1}(a)_{k-1}}\right\}^{\frac{1}{k-1}} .
$$

The result is sharp for the function $f(z)$ given by

$$
f_{k}(z)=z-\frac{2 p \beta(1-q)(1)_{k-1}(a)_{k-1}}{[(k-1)(1-m \beta)-2 p \beta(k-q)](c)_{k-1}(\lambda+1)_{k-1} B_{k}(\lambda)} \quad(k \geqslant n+1, n \in N) .
$$


P r o o f. It suffices to show that $\left|\frac{z f^{\prime}(z)}{f(z)}-1\right| \leqslant 1-\delta,|z|<r_{1}$. We have

$$
\left|\frac{z f^{\prime}(z)}{f(z)}-1\right|=\left|\frac{-\sum_{k=n+1}^{\infty}(k-1) a_{k} z^{k-1}}{1-\sum_{k=n+1}^{\infty} a_{k} z^{k-1}}\right| \leqslant \frac{\sum_{k=n+1}^{\infty}(k-1) a_{k}|z|^{k-1}}{1-\sum_{k=n+1}^{\infty} a_{k}|z|^{k-1}} .
$$

To prove the theorem, we must show that

$$
\frac{\sum_{k=n+1}^{\infty}(k-1) a_{k}|z|^{k-1}}{1-\sum_{k=n+1}^{\infty} a_{k}|z|^{k-1}} \leqslant 1-\delta .
$$

It is equivalent to

$$
\sum_{k=n+1}^{\infty}(k-\delta) a_{k}|z|^{k-1} \leqslant 1-\delta
$$

Using Theorem 1, we obtain

$$
|z| \leqslant\left\{\frac{(1-\delta)[(k-1)(1-m \beta)-2 p \beta(k-q)](c)_{k-1}(\lambda+1)_{k-1} B_{k}(\lambda)}{2 p \beta(1-q)(k-\delta)(1)_{k-1}(a)_{k-1}}\right\}^{\frac{1}{k-1}} .
$$

Hence the proof is complete.

Theorem 9. Let the function $f(z)$ of the form (1.2) be in the class $A_{n}(m, \beta, p, q, \lambda)$. Then $f$ is univalent convex of order $\delta(0 \leqslant \delta<1)$ in $|z|<r_{2}$, where

$$
r_{2}=\inf _{k}\left\{\frac{(1-\delta)[(k-1)(1-m \beta)-2 p \beta(k-q)](c)_{k-1}(\lambda+1)_{k-1} B_{k}(\lambda)}{2 p \beta k(1-q)(k-\delta)(1)_{k-1}(a)_{k-1}}\right\}^{\frac{1}{k-1}} .
$$

The result is sharp for the function $f(z)$ given by

$$
f_{k}(z)=z-\frac{2 p \beta(1-q)(1)_{k-1}(a)_{k-1}}{[(k-1)(1-m \beta)-2 p \beta(k-q)](c)_{k-1}(\lambda+1)_{k-1} B_{k}(\lambda)} \quad(k \geqslant n+1, n \in N) .
$$

P r o o f. It suffices to show that $\left|\frac{z f^{\prime \prime}(z)}{f^{\prime}(z)}\right| \leqslant 1-\delta,|z|<r_{2}$. We have

$$
\left|\frac{z f^{\prime \prime}(z)}{f^{\prime}(z)}\right|=\left|\frac{-\sum_{k=n+1}^{\infty} k(k-1) a_{k} z^{k-1}}{1-\sum_{k=n+1}^{\infty} k a_{k} z^{k-1}}\right| \leqslant \frac{\sum_{k=n+1}^{\infty} k(k-1) a_{k}|z|^{k-1}}{1-\sum_{k=n+1}^{\infty} k a_{k}|z|^{k-1}} .
$$

To prove the theorem, we must show that

$$
\frac{\sum_{k=n+1}^{\infty} k(k-1) a_{k}|z|^{k-1}}{1-\sum_{k=n+1}^{\infty} k a_{k}|z|^{k-1}} \leqslant 1-\delta .
$$

It is equivalent to

$$
\sum_{k=n+1}^{\infty} k(k-\delta) a_{k}|z|^{k-1} \leqslant 1-\delta .
$$

Using Theorem 1, we obtain

$$
\begin{aligned}
& |z|^{k-1} \leqslant\left\{\frac{(1-\delta)[(k-1)(1-m \beta)-2 p \beta(k-q)](c)_{k-1}(\lambda+1)_{k-1} B_{k}(\lambda)}{2 p \beta k(1-q)(k-\delta)(1)_{k-1}(a)_{k-1}}\right\}, \\
& |z| \leqslant\left\{\frac{(1-\delta)[(k-1)(1-m \beta)-2 p \beta(k-q)](c)_{k-1}(\lambda+1)_{k-1} B_{k}(\lambda)}{2 p \beta k(1-q)(k-\delta)(1)_{k-1}(a)_{k-1}}\right\}^{\frac{1}{k-1}} .
\end{aligned}
$$

Hence the proof is complete. 
Theorem 10. Let the function $f(z)$ of the form (1.2) be in the class $A_{n}(m, \beta, p, q, \lambda)$. Then $f$ is univalent close-to-convex of order $\delta(0 \leqslant \delta<1)$ in $|z|<r_{3}$, where

$$
r_{3}=\inf _{k}\left\{\frac{(1-\delta)[(k-1)(1-m \beta)-2 p \beta(k-q)](c)_{k-1}(\lambda+1)_{k-1} B_{k}(\lambda)}{2 p \beta k(1-q)(1)_{k-1}(a)_{k-1}}\right\}^{\frac{1}{k-1}} .
$$

The result is sharp for the function $f(z)$ given by (7.1).

P r o o f. It suffices to show that $\left|f^{\prime}(z)-1\right| \leqslant 1-\delta,|z|<r_{3}$. We have

$$
\left|f^{\prime}(z)-1\right|=\left|-\sum_{k=n+1}^{\infty} k a_{k} z^{k-1}\right| \leqslant \sum_{k=n+1}^{\infty} k a_{k}|z|^{k-1} .
$$

Thus $\left|f^{\prime}(z)-1\right| \leqslant 1-\delta$, if

$$
\sum_{k=n+1}^{\infty} \frac{k a_{k}}{1-\delta}|z|^{k-1} \leqslant 1
$$

Using Theorem 1, the above inequality holds true if

$$
|z|^{k-1} \leqslant\left\{\frac{(1-\delta)[(k-1)(1-m \beta)-2 p \beta(k-q)](c)_{k-1}(\lambda+1)_{k-1} B_{k}(\lambda)}{2 p \beta k(1-q)(1)_{k-1}(a)_{k-1}}\right\}
$$

or

$$
|z| \leqslant\left\{\frac{(1-\delta)[(k-1)(1-m \beta)-2 p \beta(k-q)](c)_{k-1}(\lambda+1)_{k-1} B_{k}(\lambda)}{2 p \beta k(1-q)(1)_{k-1}(a)_{k-1}}\right\}^{\frac{1}{k-1}} .
$$

Hence the proof is complete.

\section{REFERENCES}

1. Aghalary R., Kulkarni S. Some theorem on univalent functions, J. India Acad. Math., 2002, vol. 24, no. 1, pp. 81-93.

2. Aouf M.K. Neighborhoods of certain class of analytic functions with negative coefficients, International Journal of Mathematics and Mathematical Sciences, 2006, Article ID 38258, 6 p. http://dx.doi.org/10.1155/IJMMS/2006/38258

3. Goodman A.W. Univalent functions and nonanalytic curves, Proc. Amer. Math. Soc., 1957, vol. 8, no. 3, pp. 598-601.

4. Juma A.R.S., Zirar H. Properties of a subclass of $p$-valent functions defined by new operator $V_{p}^{\lambda}$, $A n$. Univ. Oradea, Fasc. Mat., 2014, vol. 21, no. 1, pp. 73-82.

5. Juma A.R.S., Kulkarni S.R. On univalent functions with negative coefficients by using generalized Salagean operator, Filomat, 2007, vol. 21, no. 2, pp. 173-184. http://dx.doi.org/10.2298/FIL0702173J

6. Khairnar S.M., More M. Certain family of analytic and univalent functions with normalized conditions, Acta Math. Acad. Paedagog. Nyházi. (N.S.), 2008, vol. 24, no. 3, pp. 333-344.

7. Kulkarni S.R. Some problems connected with univalent functions, Ph.D Thesis, 1981, Shivaji University, Kolhapur.

8. Kumar V., Shukla S.L. Multivalent functions defined by Ruscheweyh derivatives. II, Indian J. Pure Appl. Math., 1984, vol. 15, pp. 1228-1238.

9. Lakshminarasimhan T.V. On subclasses of functions starlike in the unit disc, J. Indian Math. Soc., New Ser., 1977, vol. 41, pp. 233-243.

10. Orhan H., Kamali M. Neighborhoods of a class of analytic functions with negative coefficients, Acta Math. Acad. Paedagog. Nyházi. (N.S.), 2005, vol. 21, no. 1, pp. 55-61.

11. Patel J., Cho N.E., Srivastava H.M. Certain subclasses of multivalent functions associated with a family of linear operators, Mathematical and Computer Modelling, 2006, vol. 43, no. 3-4, pp. 320-338.

12. Raina R.K., Srivastava H.M. Inclusion and neighborhood properties of some analytic and multivalent functions, JIPAM, J. Inequal. Pure Appl. Math., 2006, vol. 7, no. 1, paper no. 5, 6 p.

13. Ruscheweyh S. New criteria for univalent functions, Proc. Amer. Math. Soc., 1975, vol. 49, no. 1, pp. 109-115. 
14. Ruscheweyh S. Neighborhoods of univalent functions, Proc. Amer. Math. Soc., 1981, vol. 81, no. 4, pp. 521-527.

15. Silverman H. Univalent functions with negative coefficents, Proc. Amer. Math. Soc., 1975, vol. 51, no. 1, pp. 109-116.

Juma Abdul Rahman S., Department of Mathematics, University of Anbar, Al-Tameem Street, Ramadi, Iraq.

E-mail: dr_juma@hotmail.com

Abdul-Hussein Mushtaq Shaker, Department of Mathematics, University of Mustansiriyah, Baghdad, Iraq. E-mail: mushtdam8@yahoo.com

Hani Mohammad Falih, Department of Mathematics, University of Mustansiriyah, Baghdad, Iraq.

E-mail: mohfalih17@yahoo.com

\section{А. Р. С. Дәсума, М. ШІ. Абдул-Хуссейн, М.Ф. Хани \\ Об одном подклассе однолистных функций с отрицательными коэффициентами, заданном линейным оператором}

Ключевые слова: аналитические однолистные функции, произведение Адамара, производная Рушевея, теоремы искажения, теоремы о замыкании.

УДК 517.53

В работе вводится и исследуется подкласс $A_{n}(m, \beta, p, q, \lambda)$ однолистных функций с отрицательными коэффициентами, определяемый новым линейным оператором $J^{\lambda}$ в открытом единичном круге $\mathcal{U}=\{z \in \mathbb{C}:|z|<1\}$. Основной задачей является изучение следующих свойств и характеристик: оценки коэффициентов, теоремы искажения, теоремы о замыкании, окрестность функции, радиусы звездообразности, выпуклости и почти выпуклости функций, принадлежащих классу $A_{n}(m, \beta, p, q, \lambda)$.

\section{СПИСОК ЛИТЕРАТУРЫ}

1. Aghalary R., Kulkarni S. Some theorem on univalent functions // J. India Acad. Math. 2002. Vol. 24. № 1. P. 81-93.

2. Aouf M.K. Neighborhoods of certain class of analytic functions with negative coefficients // International Journal of Mathematics and Mathematical Sciences. 2006. Article ID 38258. 6 p. http://dx.doi.org/10.1155/IJMMS/2006/38258

3. Goodman A.W. Univalent functions and nonanalytic curves // Proc. Amer. Math. Soc. 1957. Vol. 8. № 3. P. 598-601.

4. Juma A.R.S., Zirar H. Properties of a subclass of $p$-valent functions defined by new operator $V_{p}^{\lambda} / /$ An. Univ. Oradea. Fasc. Mat. 2014. Vol. 21. № 1. P. 73-82.

5. Juma A.R.S., Kulkarni S.R. On univalent functions with negative coefficients by using generalized Salagean operator // Filomat. 2007. Vol. 21. № 2. P. 173-184. http://dx.doi.org/10.2298/FIL0702173J

6. Khairnar S.M., More M. Certain family of analytic and univalent functions with normalized conditions // Acta Math. Acad. Paedagog. Nyházi. (N.S.). 2008. Vol. 24. № 3. P. 333-344.

7. Kulkarni S.R. Some problems connected with univalent functions: Ph.D Thesis, 1981. Shivaji University, Kolhapur.

8. Kumar V., Shukla S.L. Multivalent functions defined by Ruscheweyh derivatives. II // Indian J. Pure Appl. Math. 1984. Vol. 15. P. 1228-1238.

9. Lakshminarasimhan T.V. On subclasses of functions starlike in the unit disc // J. Indian Math. Soc. New Ser. 1977. Vol. 41. P. 233-243.

10. Orhan H., Kamali M. Neighborhoods of a class of analytic functions with negative coefficients // Acta Math. Acad. Paedagog. Nyházi. (N.S.). 2005. Vol. 21. № 1. P. 55-61.

11. Patel J., Cho N.E., Srivastava H.M. Certain subclasses of multivalent functions associated with a family of linear operators // Mathematical and Computer Modelling. 2006. Vol. 43. № 3-4. P. 320-338. 
12. Raina R.K., Srivastava H.M. Inclusion and neighborhood properties of some analytic and multivalent functions // JIPAM. J. Inequal. Pure Appl. Math. 2006. Vol. 7. № 1. Paper № 5. 6 p.

13. Ruscheweyh S. New criteria for univalent functions // Proc. Amer. Math. Soc. 1975. Vol. 49 . № 1. P. 109-115.

14. Ruscheweyh S. Neighborhoods of univalent functions // Proc. Amer. Math. Soc. 1981. Vol. 81 . № 4. P. 521-527.

15. Silverman H. Univalent functions with negative coefficents // Proc. Amer. Math. Soc. 1975. Vol. 51. № 1. P. 109-116.

Поступила в редакцию 29.04.2015

Джума Абдул Рахман С., отделение математики, Аль-Анбар университет, Рамади, Ирак.

E-mail: dr_juma@hotmail.com

Абдул-Хуссейн Муштак Шакер, отделение математики, Аль-Мустансирия университет, Багдад, Ирак. E-mail: mushtdam8@yahoo.com

Хани Мохаммад Фалих, отделение математики, Аль-Мустансирия университет, Багдад, Ирак.

E-mail: mohfalih17@yahoo.com 